\title{
The Role of Periodontal Disease in Etiology of Myocardial Infarction
}

\author{
Stankovic $B^{1 *}$ and Minic $\mathbb{I}^{2}$ \\ ${ }^{1}$ Medical Faculty, Rihan Heights A, 0501, Abu Dhabi, UAE \\ ${ }^{2}$ Department of Periodontology and Oral medicine, Medical Faculty, University of Nis, Serbia \\ *Corresponding author: Bobana Stankovic, Medical Faculty, Rihan Heights A, 0501, Abu Dhabi, UAE, Tel: +971504127533; E-mail: \\ bobanastan85@gmail.com
}

Received date: February 12, 2019; Accepted date: March 04, 2019; Published date: March 08, 2019

Citation: Stankovic B, Minic I (2019) The Role of Periodontal Disease in Etiology of Myocardial Infarction. Arch Med S1:4

Copyright: (C2019 Stankovic B. This is an open-access article distributed under the terms of the Creative Commons Attribution License, which permits unrestricted use, distribution, and reproduction in any medium, provided the original author and source are credited.

\section{Abstract}

Periodontal disease is a chronic inflammatory disease of periodontium and its advanced form is characterized by periodontal ligament loss and destruction of surrounding alveolar bone. Cardiovascular diseases are the most common cause of adult death in the world. Over $80 \%$ of cardiovascular disease deaths take place in low-and middleincome countries and occur almost equally in men and women. The main causes of cardiovascular diseases are tobacco use, unhealthy diet, physical inactivity, high blood pressure and high blood cholesterol. The association of coronary heart disease and periodontal disease may be due to an underlying response trait, which places an individual at high risk for developing both periodontal disease and atherosclerosis. It was suggested that periodontal disease, once established provides a biological burden of endotoxin and inflammatory cytokines, especially thromboxane $A 2$, prostaglandin $\mathrm{E} 2$, interleukin (IL) $1 \mathrm{~L}=1 \alpha$, and tumor necrosis factor- $\beta$, which serve to initiate and exacerbate atherogenesis and thromboembolic events. As we known dental infection especially periodontal disease can be tightly connected with cardiovascular disease, that's the reason why patients who have valvular defect or some other congenital defects such as septal defects, or who have prosthetic valve, should receive antibiotic therapy as prophylaxis before any blood included procedure. It is very important to prevent periodontal disease, or if it's already present, the treatment should commence as soon as possible to prevent the risk factors from occurring and causing cardiovascular disease.

Keywords: Periodontal disease; IL-1; TNF- $\alpha$; CRP; Cardiovascular diseases

\section{Introduction}

Periodontal disease is a chronic inflammatory disease of periodontium and its advanced form is characterized by periodontal ligament loss and destruction of surrounding alveolar bone [1]. It is the main cause of tooth loss and is considered one of the two biggest threats to the oral health $[1,2]$.
The initiation and progression of periodontal disease depend on the presence of pathogenic bacteria, host response and risk factors. Dental biofilm, also known as plaque, develops and matures over a period of several weeks and consists mainly of anaerobic bacteria. Over time flora changes due to presence of specific bacteria in patients with periodontal disease: $A$. actinomycetemcomitans, $P$. gingivalis, $T$. forsythia (B. forsythus), P. intermedia, Fusobacterium nucleatum and Peptostreptococcus micros. The scientists concluded that the presence of these bacteria is a marker for destructive periodontal disease [3].

The most influential factors are: oral hygiene, race, socioeconomic status, age, stress, smoking, use of medications and alcohol and drug abuse. Based on a recent longitudinal study spanning 26 years, the investigators concluded that the two factors most predictive of periodontal disease progression were smoking and increased levels of calculus [4].

Periodontal disease is the most common oral condition of human population [5]. Periodontal disease describes a group of inflammatory conditions that affect the supporting structures of the teeth or periodontium. The initiation, development, diagnosis, and subsequent treatment of periodontal disease are today very well known. Microbal plaque is generally considered to be the initiating factor in periodontal disease.

When plaque accumulates on tooth and gingival surfaces, it instigates the development of an inflammatory response are critical to the clinical outcome. If the inflammatory response is sufficient to control the challenge from plaque without destruction of periodontal ligament or alveolar bone, the clinical condition is termed gingivitis. If there is destruction of periodontal ligament and alveolar bone, the condition is termed periodontitis. The fundamental diagnosis of gingivitis or periodontitis affects treatment.

Most of studies who have published about periodontal diseases in the Arab world have been carried out in school children and adolescents. General finding from the studies reviewed is that periodontitis is more prevalent among older age groups. Furthermore, studies on the reasons of tooth extractions in the Arab countries show that more than one third of missing permanent teeth have been extracted because of 
periodontitis especially for those who are older than 40 years [6].

Cardiovascular diseases are the most common cause of adult death in the world. Over $80 \%$ of cardiovascular disease deaths take place in low-and middle-income countries and occur almost equally in men and women. The main causes of cardiovascular diseases are tobacco use, unhealthy diet, physical inactivity, high blood pressure and high blood cholesterol $[7,8]$. It is a wellknown fact that cardiovascular disease are the top killer in the $\mathrm{UAE}$, causing about 30 per cent of all deaths in the country.

These diseases are also occurring among more and more young patients, and their presentation is quite complex among some of the most at-risk groups. In the UAE, about 20 per cent of the adult population smokes, and this is one of the biggest factors contributing to cardiovascular risk among people from the Arab region.

At the same time, people from the Indian subcontinent and the UAE are already more prone to cardiovascular disease, being afflicted a decade earlier then their Western counterparts. The UAE's record with cardiovascular disease mirrors global trends, with more than 17.5 million people dying each year across the world from cardiovascular diseases, according to the World Health Organization. The risk of cardiovascular diseases arises due to unhealthy lifestyles, as well as the high prevalence of diabetes, obesity, hypertension, hypercholesterolemia and smoking.

The most common cardiovascular diseases are: myocardial infarction, coronary artery disease, heart failure, rheumatic fever, cardiomyopathy, congenital heart defect, cardiac arrest etc.

Myocardial infarction commonly known as a heart attack is a life-threatening condition that occurs when the portion of the heart is deprived of oxygen due to blockage of a coronal artery by plaque. Blood flow decreases or stops, causing damage to heart muscle. Symptoms of a heart attack include: chest pain or discomfort in the center of the chest, described as heaviness, tightness, burning, pressure that lasts for more than a few minutes or goes away and comes back, than pain in the upper part of the body such as arms, left shoulder, neck, jaw or stomach, sweating, difficulty in breathing or shortness of breath, nausea or vomiting, rapid or irregular heartbeats. Myocardial infection can occur among all patients, though it is more common among women or people with diabetes $[9,10]$. Consequences of untreated myocardial infarction are: arrhythmias (most common immediate cause of death), congestive heart failure, myocardial rupture, which may result in death from cardiac tamponed, thrombus formation on infarcted tissue; may result in systemic embolism and death.

The association of coronary heart disease and periodontal disease may be due to an underlying response trait, which places an individual at high risk for developing both periodontal disease and atherosclerosis. It was suggested that periodontal disease, once established provides a biological burden of endotoxin and inflammatory cytokines, especially thromboxane $\mathrm{A} 2$, prostaglandin $\mathrm{E} 2$, interleukin (IL) $1 \mathrm{~L}=1$, and tumor necrosis factor- $\beta$, which serve to initiate and exacerbate atherogenesis and thromboembolic events [11].

\section{Interleukin-1}

Interleukin-1 was first described in 1972 as a lymphocyteactivating factor, but later was established that interleukin-1 has big role in induction of inflammation, stimulation of proliferation of $\mathrm{T}$ and $\mathrm{B}$-cells, induction of acute phase proteins and prostaglandin or regulation of hematopoiesis. It can also be involved in regulation of blood calcium levels, regulation of blood pressure or modulation of sleep. Its activity is very important in pathogenesis of cardiovascular diseases, mainly due to its pro-inflammatory potential. Certain IL-1 gene variations have been associated with overexpression of inflammatory mediators and also with increased risk of cardiovascular events [12].

The polypeptide hormone interleukin (IL-1) is one of the key mediators of the body's response to microbial invasion, inflammation, immunological reactions, and tissue injury. IL-1 is a prominent member of a group of polypeptide mediators now called cytokines. In the first hours after infection or injury, the biological effects of IL-1 are manifested in nearly every tissue and organ. Unlike other interleukins (interleukins 2-6), IL-1 is not a newly discovered molecule. In 1940's this material was called endogenous pyrogen for its ability to produce fever [13].

\section{Tumor necrosis factor}

Tumor necrosis factor alpha (TNF-) was originally identified as a factor that leads to rapid necrosis of transplantable tumors in mice [14] and now it is considered a pro-inflammatory cytokine involved in the innate immune response [15]

Tumor necrosis factor-is a cell signaling protein (cytokine), produced by white blood cells. Macrophages are the major producers of TNF-and interestingly are also high responsive to TNF-. Its substantial roll in periodontitis mediated bone loss is well known [16]. It can be detected in saliva, and gingival cervical fluid, in both health and periodontitis [17].

TNF factor was identified as the mediator of the cardiac inflammatory responses in patients who have acute myocardial infarction $[18,19]$. Actually, myocardial TNF is very important contributor to myocardial contractile dysfunction and cardiomyocyte death post myocardial infarction and it take part of roll in chronic heart failure $[20,21]$.

\section{C- reactive protein (CRP)}

C-reactive protein(CRP), an acute phase protein from pentraxin family of proteins, seen in blood in growing concentration in case of an injury, inflammation or tissue death. CRP is one of the common test parameters used in clinical practice to prognose inflammation (also known as a biomarker of inflammation), and can be viewed as a direct contributor in atherosclerosis as it functions both as' pro-inflammatory' and 'anti-inflammatory' molecule [22]. 
The protein has emerged as one of the most powerful independent predictors of cardiovascular disease. CRP level, which is always significantly high in acute coronary syndromes, which has a prognostic value in patients with cardiovascular complications [23].

Many studies have found close association between chronic periodontitis and C-reactive protein. Periodontal disease involves chronic inflammatory processes resulting from interaction of selected gram negative bacteria with the host defense in disease susceptible individuals. The host responds to the microbial challenge, with a high inflammatory response with increased levels of cytokines. These mediators promote activation of the acute phase reactants resulting in elevated serum levels of CRP [24].

\section{Conclusion}

Many patients with heart disease require dental treatment. Various surveys have suggested that dental disease may possibly contribute to the development of atherosclerosis and myocardial infarction. As we known dental infection especially periodontal disease can be tightly connected with cardiovascular disease, that's the reason why patients who have valvular defect or some other congenital defects such as septal defects, or who have prosthetic valve, should receive antibiotic therapy as prophylaxis before any blood included procedure [25].

Risk factors are certain conditions that increase a person's risk for cardiovascular disease. Non modifiable risk factors such as increasing age, male gender, menopause, family history, and race are risk factors that cannot be changed, while modifiable risk factors can be modified, controlled, or treated. The more risk factors we have the greater our chance developing cardiovascular disease [26].

It is very important to prevent periodontal disease, or if it's already present, the treatment should commence as soon as possible to prevent the risk factors from occurring and causing cardiovascular disease.

\section{References}

1. De Pablo P, Chapple IL, Buckley CD, Dietrich T (2009) Periodontitis in systemic rheumatic diseases. Nat Rev Rheumatol 5: 218-224.

2. Benjamin RM (2010) Oral health: The silent epidemic. Public Health Rep 125: 158-159.

3. Van Winkelhoff AJ, Loos BG, van der Reijden WA, van der Velden $U$ (2002) Porphyromonas gingivalis, Bacteroides forsythus and other putative periodontal pathogens in subjects with and without periodontal destruction. J Clin Periodontol 29: 1023-1028.

4. Tracy RP (2003) Thrombin, inflammation and cardiovascular disease. Chest. 124: 49S-57S.

5. Blencowe $\mathrm{H}$, Cousens $\mathrm{S}$, Oestergaard $\mathrm{M}$, Chou $\mathrm{D}$, Moller $\mathrm{AB}$, et al. (2012) National, regional and worldwide estimates of preterm birth. The Lancet 379:2162-2172.

6. Raitapuro-Murray T, Molleson TI, Hughes FJ (2014) The prevalence of periodontal disease in a Romano-British population c 200-400 AD. Br Dent J 217: 459-466.
7. Haddad I, Haddadin K, Jebrin S (1999) Reasons for extraction of permanent teeth in Jordan. Int Dent J 49: 343-346.

8. Hassan AK (2000) Reasons for tooth extraction among patients in Sebha, Libyan Arab Jamahiriya: a pilot study. East Mediterr Health J 6: 176-178.

9. Al-Shammari KF, Al-Ansari JM, Al-Melh MA (2006) Reasons for tooth extraction in Kuwait. Med Princ Pract 15: 417-422.

10. Brinson CW, Lu Z, Li Y, Lopes-Virella MF, Huang Y (2016) Lipopolysaccharide and IL-1ß coordinate a synergy on cytokine production by upregulating MyD88 expression in human gingival fibroblasts. Mol Immunol 79: 47-54.

11. Goldenberg R, Culhane J, lams J, Romero R (2008) Epidemiology and causes of preterm birth. The Lancet 371: 75-84.

12. Boyce BF, Li P, Yao Z, Zhang Q, Badell IR, et al. (2005) TNF-alpha and pathologic bone resorption. Keio J Med 54: 127-131.

13. Neumann FJ, Ott I, Gawaz M, Richardt G, Holzapfel H, et al. (1995) Cardiac release of cytokines and inflammatory responses in acute myocardial infarction. Circulation 92: 748-755.

14. Frangogiannis NG, Lindsey ML, Michael LH, Youker KA, Bressler RB, et al. (1998) Resident cardiac mast cells degranulate and release preformed TNF-alpha, initiating the cytokine cascade in experimental canine myocardial ischemia/reperfusion. Circulation 98: 699-710.

15. Ping Z, Aiqun $M$, Jiwu L, Liang $S$ (2017) TNF Receptor $1 / 2$ Predict Heart Failure Risk in Type 2 Diabetes Mellitus Patients. Int Heart J 58: $245-249$

16. Meldrum DR, Cleveland JC, Cain BS, Meng X, Harken AH (1998) Increased myocardial tumor necrosis factor-alpha in a crystalloid perfused model of cardiac ischemia-reperfusion injury. Ann Horac Surg 65: 439-443.

17. Pepys MG and Hirsch eld GM (2003) C-reactive protein: A critical update. J Clin Invest 111: 12

18. Boncler M, Luzak B, Watala C (2006) Role of C-reactive protein in artherogensis. Postepy Hig Med Dosw 60: 538-546.

19. Joseph R, Narayan V, Krishnan R, Sreelatha M (2011) Non-Surgical periodontal therapy improves serum levels of C-reactive protein and edematous states in female patients with idiopathic edema. $J$ periodontal 182: 201-209.

20. Helmer $\mathrm{H}$, Tretzmüller $\mathrm{U}$, Brunbauer $\mathrm{M}$, Kaider $\mathrm{A}$, Husslein $\mathrm{P}$, et al. (2002) Production of oxytocin receptor and cytokines in primary uterine smooth muscle cells cultivated under inflammatory conditions. J Soc Gynecol Investig 9: 15-21.

21. Nabet C, Lelong N (2010) Maternal periodontitis and the causes of preterm birth: the case-control EPIPAP study. J Clin Periodontol 37 37-45.

22. Gürsoy M, Kononen E, Gursoy UK, Tervahartiala T, Pajukanta R, et al. (2010) Periodontal status and neutrophilic enzyme levels in gingival crevicular fluid during pregnancy and postpartum. J Periodontol 81: 1790-1796.

23. Choi BK, Moon SY, Cha JH, Kim KW, Yoo YJ (2005) Prostaglandin $E(2)$ is a main mediator in receptor activator of nuclear factorkappaB ligand-dependent osteoclastogenesis induced by Porphyromonas gingivalis, Treponema denticola, and Treponema socranskii. J Periodontol 76: 813-820.

24. Van Dyke TE, van Winkelhoff AJ (2013) Infection and inflammatory mechanisms. J Clin Periodontol 40: S1-S7. 
25. Xiao LM, Yan YX, Xie CJ, Fan WH, Xuan DY, et al. (2009) Association among interleukin-6 gene polymorphism, diabetes and periodontitis in a Chinese population. Oral Dis 15: 547-553.
26. Dörtbudak O, Eberhardt R, Ulm M, Persson GR (2005) Periodontitis, a marker of risk in pregnancy for preterm birth. J Clin Periodontol 32: 45-52. 